\section{Relação entre enfermeiros e médicos em hospital escola: a perspectiva dos médicos}

\section{Professional relationship between nurses and doctors at the hospital of medical school: the view of doctors}

\begin{abstract}
The conflict between physicians and nurses, historically the two main groups of professionals responsible for patient care, is originated from the combination of several factors ranging from the establishment of the multidisciplinary team to salary issues. This study aims to determine, in the view of physicians, the existence of conflict in the relationship between them and nurses at the Hospital das Clinicas, Universidade Federal de Goiás (HC / UFG) and what factors are associated with it. To this goal, 30 doctors completed a questionnaire with demographic data and questions about the variables that affect the relationship, using a Lickert scale. Among the results, the average of age is 42.7 years, $50 \%$ of participants are female and $93.3 \%$ of the respondents worked elsewhere. The average of years of working in the HC is 16.3. Concerning to the conflict, we highlighted four factors of conflict prevention (Pc), with Middle Ranking (RM) greater than 3.0, and two others generators of conflict (2Gc). These factors are: Interprofessional Communication $\quad(R M=3.03)$; Autonomy in Team (RM=3.63); Interprofessional Relationship $(R M=3.36) ; \quad$ Working Conditions $(R M=2.26)$, Influence of Hospital as a School $(R M=2.83)$ and Patient Benefits $(R M=3,93)$. We concluded that in the analyzed reality the conflict is considered non-existent, because there is a favorable ratio of protective factors ( $4 P c: 2 G c)$, but it is imminent, due to the persistence of factors that can unleash it ("Working Conditions" and "Influence of Hospital as a School "). Moreover, there is power struggle with nursing, which can unbalance the situation and create ethical problems.
\end{abstract}

Key words Physician, Nurse, Conflict, Ethics, Relationship
Ana Maria de Oliveira 1

André Moreira Lemes 2

Carolina Rocha Machado 3

Fernanda Loyola e Silva 4

Fernanda Souza Miranda 5
1 Programa de Doutoramento em Bioética. Faculdade de Medicina. Universidade do Porto. Alameda Prof. Hernâni Monteiro, 4200319. Porto, Portugal. E-mail: dr.amoliveira@gmail.com 2-5 Universidade Federal de Goiás. Goiânia, GO, Brasil.

\section{Resumo}

$O$ conflito entre profissionais da medicina e da enfermagem, historicamente as duas principais categorias profissionais responsáveis pelo cuidado do paciente, é originado a partir da associação de diversos fatores que vão desde a constituição da equipe multiprofissional até as questões salariais. $O$ presente estudo pretende verificar se, na visão dos médicos, existe conflito na relação entre médicos e enfermeiros no Hospital das Clínicas da Universidade Federal de Goiás (HC/UFG) e quais são os fatores associados. Para tanto, 30 médicos responderam a um questionário com dados demográficos e perguntas em escala Lickert, sobre as variáveis que interferem na relação interprofissional. Dentre os resultados, a média de idade é de 42,7 anos, 50\% dos participantes é do sexo feminino e 93,3 dos entrevistados trabalha em outro lugar. A média de anos de trabalho no HC é de 16,3. Quanto às categorias de conflitos se destacam quatro fatores preventivos de conflitos (Pc) com Ranking Médio (RM) maior que 3,0 e dois geradores de conflito (2Gc), quais sejam: Comunicação interprofissional (RM =3,03); Autonomia na equipe $(R M=3,63) ;$ Relação interprofissional $(R M=3,36)$; Condições de trabalho ( $R M=2,26)$; Influência do hospital escola $(R M=2,83)$ e Reflexo no paciente (RM=3,93). Conclui-se que na realidade analisada o conflito é inexistente, por haver uma relação favorável de fatores protetores (4Pc:2Gc), porém o mesmo é iminente, devido à permanência de fatores que podem desencadeá-lo ("Condições de trabalho" e "Influência do hospitalescola"). Além disso, há disputa de poder com a enfermagem, o que pode desequilibrar a situação $e$ gerar problemas éticos.

Palavras-chave Médico, Enfermeiro, Conflito, Ética, Relação 


\section{Introdução}

$\mathrm{Na}$ complexa estrutura das organizações hospitalares interagem duas categorias profissionais de grande importância na execução das atividades inerentes a essas instituições: médicos e enfermeiros. Por atuarem de forma interdependente, mantém-se em estreito vínculo e com um potencial para desencadear conflitos interprofissionais.

Considerando as peculiaridades da equipe multidisciplinar em saúde, Silva ${ }^{1}$ define os médicos e enfermeiros como grupos de maior representatividade para os serviços nessa área, apontando ainda a relação entre esses grupos como conflitante. Considera-se que os problemas advindos desse relacionamento atuam como importante obstáculo à excelência na produção em serviços de saúde. ${ }^{2}$

A construção da relação dicotômica médicoenfermeiro abrange atributos históricos de cada ator da organização social instituída como equipe multidisciplinar em saúde, bem como contempla a noção de representações sociais dos mesmos.

O conflito entre médico e enfermeiro é um dos principais problemas nas instituições de saúde, já que entre eles se estabelece o mais estreito vínculo profissional. Desse conflito, emergem problemas que prejudicam a relação em uma equipe multidisciplinar, e, principalmente, o paciente, cujos cuidados resultam dessa relação. ${ }^{3}$ Tal conflito, muitas vezes velado, é traduzido sob a forma de uma discreta disputa de poder, em que ambos querem demonstrar o seu papel preponderante no tratamento do doente: o que prescreve ou o que administra os medicamentos. O primeiro se recusa a ouvir os conselhos do segundo, que por sua vez se recusa a obedecer às recomendações do primeiro. Isso implica em perturbações no seguimento de esquemas terapêuticos, ou mesmo em erros que poderiam ser evitados.

Vários autores 3,4 afirmam o quão marcante é o papel do médico na definição do papel do enfermeiro, e quão interessante e controversa é a identidade construída com base em tal relação conflituosa.

A percepção é um importante processo psicológico que influencia os relacionamentos humanos. Ela contribui para o conhecimento que vai se formando sobre a realidade objetiva. Silva (2006: 86) 1 afirma que "embora baseada em processos sensoriais, ela se caracteriza por sua natureza subjetiva, pois sobre ela recaem variáveis psicológicas, tais como: características de personalidade, valores, crenças, hábitos, estados emocionais, história pessoal".

Delineada sob a forma de equipe multiprofissional, a divisão do trabalho em unidade hospitalar surge como forma de enfrentamento da complexidade inerente às peculiaridades do sistema de saúde, à dinâmica do processo saúde-doença e à dimensão holística do cuidar do paciente. Peduzzi ${ }^{5}$ afirma que o ideário acerca da equipe multidisciplinar configura-se como estratégia para enfrentar o intenso processo de especialização na área da saúde.

Pinho $^{6}$ esclarece que o trabalho em equipe origina-se a partir da necessidade de promover a qualidade dos serviços em saúde, de maneira a atender eficientemente às demandas do paciente.

As divergências de opiniões e condutas são naturais e inevitáveis no local de trabalho. O trabalho em equipe possui três justificativas principais, a saber:7 a) quebra da divisão do processo de trabalho; b) possibilidade de responsabilização de cada equipe por um conjunto de problemas bem delimitados; e c) possibilidade de superação da inércia e da indiferença burocrática que caracterizam os serviços públicos de saúde atualmente. As relações conflituosas podem propiciar, frequentemente, o (re)delineamento e a (re)definição de papéis, de modo a alcançar a adaptação da equipe às demandas da assistência em saúde. 1

Para os teóricos interacionistas, os conflitos são uma necessidade absoluta e um estímulo às organizações, no sentido de gerar crescimento. Podem apresentar caráter construtivo ou destrutivo, de acordo com o modo como são gerados $\mathrm{e}$ conduzidos. 8

Segundo a formulação teórica de Peduzzi (2001: $103)^{5}$ "trabalho em equipe consiste numa modalidade de trabalho coletivo que se configura na relação recíproca entre as intervenções técnicas e a interação dos agentes. A tipologia proposta refere-se a duas modalidades de equipe: equipe integração e equipe agrupamento, e os critérios de reconhecimento dos tipos de equipe dizem respeito à comunicação entre os agentes do trabalho; diferenças técnicas e desigual valoração social dos trabalhos especializados; formulação de um projeto assistencial comum; especificidade de cada área profissional; e flexibilidade da divisão do trabalho e autonomia técnica".

Optou-se pela denominação enfermeiros e médicos para se referir aos profissionais sem atender à distinção de gênero. No entanto, isso não significa que os autores ignorem as questões que permeiam as relações profissionais.

O presente trabalho visa a identificar e caracterizar o conflito na relação entre médicos e enfermeiros, na perspectiva do profissional médico, a partir da realidade observada no Hospital das Clínicas da Universidade Federal de Goiás e dar 
oportunidade a reflexão no sentido do caráter formador e construtivo do conflito nos aspectos éticos da relação profissional.

\section{Métodos}

O Hospital das Clínicas da Universidade Federal de Goiás (HC/UFG), segundo dados do setor de recursos humanos, possui em seu quadro de servidores 411 médicos com variados tipos de vínculos: Ministério da Saúde, Secretaria Estadual de Saúde (SES) e Secretaria Municipal de Saúde (SMS), Universidade (UFG) e voluntários. Possui 108 docentes médicos da Faculdade de Medicina da UFG e de outras instituições que realizam atividades assistenciais nas enfermarias e ambulatórios.

Foram entrevistados 30 médicos servidores do $\mathrm{HC} / \mathrm{UFG}$, durante os períodos da manhã, tarde e noite, perfazendo uma amostra de $16 \%$ do total de médicos alocados no $\mathrm{HC}$, sendo a amostra representativa ( $\mathrm{n}>15$, intervalo de confiança de $95 \%$ ).

Como instrumento de avaliação utilizou-se um questionário padronizado com as respostas escalonadas, conforme a escala de Likert, 3 além de informações sobre aspectos pessoais e profissionais: idade, sexo, tempo de trabalho no hospital, carga horária semanal de trabalho e atividades de docência. Os dados obtidos foram tratados estaticamente com o auxílio do programa Epi Info versão 3.4.3 e feita a análise descritiva e fatorial por meio de ranking médio (RM)

Os itens da escala de Likert assumiram os seguintes valores: nunca equivalendo a zero; raramente, um; às vezes, 2; quase sempre, 3; sempre, 4. Para análise fatorial, as perguntas em escala de Likert foram agrupadas em categorias seguindo padrões descritos na literatura. ${ }^{3} \mathrm{~A}$ assertiva 1 correspondeu à categoria "comunicação interprofissional"; de 2 a 5 à "autonomia na equipe"; 6 e 7 à "relação interprofissional"; de 8, 9 e 10, "condições de trabalho"; 11 e 12, "influência do hospital-escola" e 13 "reflexo no paciente" da relação entre médicos e enfermeiros. Foi questionado também se havia disputa de poder com a enfermagem (questão 15) e se esta interferia em problemas éticos (questão 16). Após a análise fatorial RM adotou-se ponto de corte igual a 3 , sendo definido que, se a média da categoria fosse igual ao superior a 3 (três), consideravase o fator preventivo para o conflito $(\mathrm{Pc})$. Caso contrário, ou seja, se a média for menor que 3 , considerava-se que aquele fator seria gerador de conflito (Gc). Assim, uma vez que o número de fatores geradores de conflito era maior que o de fatores de prevenção $(\mathrm{Gc}>\mathrm{Pc})$, considerava-se que havia conflito estabelecido na realidade estudada. Se a análise resultasse em número de fatores geradores de conflito menor que o de prevenção $(\mathrm{Gc}<\mathrm{Pc})$, considerava-se que na realidade não há conflito instalado, porém iminente. Se o número de fatores geradores de conflito fosse nulo $(\mathrm{Gc}=0)$, considerava-se que a realidade analisada estava protegida do conflito naquele instante.

\section{Resultados}

A análise revelou que a média de idade foi de 42,7 anos, sendo que o participante mais jovem tem $27 \mathrm{e}$ o mais velho 61. A distribuição por sexo foi de $50 \%$ dos participantes de cada sexo. Verificou-se que quase a totalidade dos entrevistados $(93,3 \%)$ trabalha em outro lugar. A média de anos de trabalho no HC é de 16,3, com o tempo mínimo foi menor que um ano e o máximo 36 anos. A média da carga horária semanal de trabalho foi de 51,6 horas, com moda de 60 horas $(40,7 \%)$, e a carga horária semanal no $\mathrm{HC}$ foi de 31,8 horas, com moda de 40 horas $(33,3 \%)$.

Quanto ao exercício da docência, 15 entrevistados eram professores, sendo 11 da Faculdade de Medicina.

Quanto ao salário, 79,3\% dos entrevistados afirmaram que o mesmo não é condizente com as atividades realizadas.

$\mathrm{Na}$ categoria "comunicação interprofissional" o RM foi de 3,03, revelando que há comunicação eficaz entre os profissionais (enfermagem e o medicina), o que representa um fator preventivo para o conflito $(\mathrm{RM}>3)$. Quanto a "autonomia na equipe" o RM foi de 3,63, revelando que na perspectiva dos médicos, os mesmos possuem autonomia na tomada de decisão acerca do cuidado do paciente $(\mathrm{RM}=3,63)$ e que as opiniões são acatadas pelos enfermeiros na maioria das ocasiões $(\mathrm{RM}=3,56)$. A divisão de tarefas na equipe multiprofissional foi adequada $(\mathrm{RM}=3,4)$ e os respondentes sabiam qual é o papel de cada um no serviço ( $R M=3,93)$.

A categoria "relação interprofissional" configurou-se como um fator preventivo para o conflito, com $\mathrm{RM}$ de 3,36 $(\mathrm{RM} \geq 3)$. Os médicos entrevistados afirmaram que existe relação profissional harmoniosa com enfermeiro e com os demais profissionais de saúde ( $\mathrm{RM}=3,43 ; \mathrm{RM}=3,33, p>0,05)$.

A maioria dos entrevistados considerou que ambiente e materiais de trabalho não são adequados - RM de 2,26 $(\mathrm{RM}<3)$, sendo considerado pelos médicos que condições adequadas de trabalho favorecem a boa relação tanto com os enfermeiros $(\mathrm{RM}=3,33)$ quanto com outros profissionais de 
saúde $(\mathrm{RM}=3,43)$.

O item "influência do hospital escola" apresenta RM de 2,83, sendo portanto, um fator gerador de conflito.

A maioria entrevistada possuia a percepção de que a boa relação entre os profissionais favorece o cuidado com o paciente ( $\mathrm{RM}=3,93 ; \mathrm{Pc})$.

Quanto à disputa de poder com a enfermagem, $73,3 \%$ dos entrevistados afirmaram que ela existe em algum momento da relação interprofissional, sendo que $90,9 \%$ destes consideraram que essa disputa propicia problemas éticos entre as categorias profissionais.

A Tabela 1 resume os achados nas categorias onde se destacam quatro fatores preventivos de conflitos e dois geradores de conflito (4Pc:2Gc), revelando que na realidade analisada o conflito é iminente, devido à existência de fatores que podem desencadeá-lo ("condições de trabalho" e "influência do hospital-escola").

Tabela 1

Categorias de conflito na perspectiva dos profissionais médicos do Hospital das Clínicas da Universidade Federal de Goiás.

\begin{tabular}{lcc}
\hline Categoria & Ranking médio & Relação com o conflito \\
\hline Comunicação interprofissional & 3,03 & PC \\
Autonomia na equipe & 3,63 & PC \\
Relação interprofissional & 3,36 & PC \\
Condições de trabalho & 2,26 & $\mathrm{GC}$ \\
Influência do hospital-escola & 2,83 & $\mathrm{GC}$ \\
Reflexo no paciente & 3,93 & $\mathrm{PC}$
\end{tabular}

$\mathrm{PC}=$ fator preventivo para o conflito $(\mathrm{RM} \geq 3) ; \mathrm{GC}=$ fator gerador de conflito $(\mathrm{RM}<3)$.

\section{Discussão}

O trabalho em saúde se caracteriza, nos tempos atuais, por ser institucional e coletivo, e busca o entendimento e reconhecimento recíproco de autoridade e de saberes, além de autonomia técnica. 9 Desde a criação do Sistema Único de Saúde (SUS), os serviços de saúde se organizaram na forma de trabalho em equipe multiprofissional, 5 com ênfase na hierarquização da atenção. Dessa forma, a partir da década de 1980 tem havido o interesse pela investigação científica na temática, com um destaque especial para os aspectos éticos/bioéticos. 10

Dentre os resultados encontrados quanto aos dados demográficos e profissiográficos, destaca-se que os médicos do HC são adultos jovens e com alta sobrecarga semanal de trabalho. Esses achados são corroborados por aqueles do Conselho Federal de Medicina, durante pesquisa realizada em 2004.11

$\mathrm{O}$ tempo médio de trabalho no $\mathrm{HC}$ foi cerca de 16 anos, porém sem associação com fatores desencadeantes do conflito. No estudo de Silva et al., ${ }^{3}$ o tempo de serviço para os médicos foi importante para análise do relacionamento médico-enfermeiro, pois, o maior tempo de convivência na organização leva a sustentação de padrões cristalizados da cultura organizacional estabelecida, acirrrando a disputa pelo poder e facilitando problemas éticos.

Quanto à tipologia das equipes multiprofissionais pode-se concluir, que a equipe multiprofissional do HC, na visão dos médicos, possui características de equipe tipo "agrupamento", por haver justaposição das ações e conexão entre os seus membros e também de equipe tipo "integração" que se caracteriza pela articulação das ações e a interação dos participantes. ${ }^{5,6,8} \mathrm{Em}$ ambos os tipos, a multidisciplinaridade encontra-se permeada por questões como hierarquização na divisão do trabalho, desigualdade na valorização social dos trabalhos distintos, diferenças técnicas e exercício da autonomia profissional.5,12,13

Lima e Bastos ${ }^{2}$ acrescentam que, de permeio aos aspectos já elencados, se adicionam outros fatores desencadeadores de conflitos, tais como: a falta de clareza na delimitação de papéis, as disputas de poder, a estrutura hospitalar verticalizada e burocratizada e a falta de comprometimento de muitos profissionais.

À semelhança dos resultados descritos por Pinho, 6 também se constatou que a grande maioria dos respondentes afirma que o paciente é beneficiado quando há boa relação entre os profissionais 
responsáveis pelo seu cuidado. Este fato configurase como prerrogativa de excelência para que cada profissional se empenhe para estabelecer relação harmoniosa com os demais profissionais. O prazer do trabalho em enfermagem está na melhora do paciente, na sensação do trabalho cumprido e o desprazer está relacionado à organização e às condições de trabalho. ${ }^{14}$

A análise fatorial permitiu avaliar os fatores que interferem na relação médico-enfermeiro, sendo que destes configuram-se como fatores de prevenção do conflito, a saber: "comunicação interprofissional", "autonomia na equipe", relação interprofissional e "reflexo no paciente". Em contrapartida, são consideradas geradoras do conflito as categorias: "influência do hospital-escola" e "condições de trabalho".

Quando se considera a literatura em geral,1,2,3,5 é recorrente a presença de conflitos entre essas duas categorias profissionais. No entanto, Zoboli10 cita pesquisa de Udén et al. (1992) a qual revela que os médicos são frequentemente apontados pelos enfermeiros como fontes de conflitos éticos; por outro lado, estes raramente são mencionados nas narrativas dos primeiros. Foi o que se constatou na realidade do HC, na visão do médico, ou seja, que não há conflito consolidado com a categoria de enfermeiros, ou mesmo com os demais profissionais envolvidos no cuidado do paciente, destacando-se que são fatores geradores de conflitos, as condições de trabalho e a fato da instituição ser um hospitalescola.

A comunicação é um outro ponto crucial para o estabelecimento de relações apropriadas em qualquer ambiente em que se necessita consolidar um grupo, 3,5,12 e diferentemente do que diz a literatura, o estudo apresenta situação onde predominam fatores protetores de conflito. Em pesquisa realizada nessa mesma instituição, Oliveira et al. 15 entrevistaram os profissionais da enfermagem, e na visão destes, a comunicação, o reconhecimento interprofissional e as condições de trabalho representam os fatores geradores de conflitos na relação com os médicos, enquanto os demais fatores foram considerados protetores de conflitos (comunicação interprofissional; divisão de tarefas e reflexo no paciente)

Mallory 13 enumera possíveis causas de divergências no trabalho em equipe, relacionadas com problemas na comunicação entre os profissionais, que, juntamente com a deficiência de materiais, de equipamentos e de pessoal são geradores de dificuldades, 4 fato este comprovado nesta pesquisa. Assim, sugere-se que a apropriada troca de informações, seguida do entendimento entre as partes e a aplicação do estabelecido e acordado consegue superar os problemas e evitar a emergência do conflito.

O estudo da cultura organizacional compreende o tecido simbólico sobre o qual a dimensão interpessoal se constitui e que está permeado pela compreensão das relações de poder, do processo de trabalho e das práticas administrativas. $\mathrm{O}$ arcabouço teórico desse campo científico, abordado pelos autores contemporâneos, parece indicar saídas possíveis para minimizar a dinâmica cotidiana desse tipo de organização. 16

Uma fonte significativa de estresse no exercício da enfermagem está no conflito com profissionais médicos. Existe evidencia na literatura que diferentes maneiras de resolver conflitos geram mais ou menos estresse. E que o nível hierárquico, a posição profissional e a reputação do enfermeiro afetam tanto a escolha estratégica de resolução de conflito quanto o nível de satisfação e de estresse no trabalho. 17

Estudo com médicos e enfermeiros em unidade de cuidados intensivos resultou que para $73 \%$ dos médicos a comunicação com enfermeiros era boa e colaborativa, enquanto que apenas $33 \%$ dos enfermeiros consideravam a alta ou muito alta a comunicação e a colaboração com médicos. 18 Por outro lado, na situação em que há disputa de poder com a enfermagem, há risco de haver desequilíbrio entre os fatores protetores e geradores de conflitos e a consequente instalação de situações de conflitos, gerando problemas éticos. Para Teixeira et al.19 falha na comunicação entre profissionais de saúde em centros de tratamento intensivo pode estar relacionado com aumento da mortalidade dos pacientes criticamente doentes.

Gracia $^{20}$ argumenta que conflitos éticos são todas as situações em que há conflitos de valores e para as quais se aplica o "sentido moral: ou seja, princípios justificáveis pelas circunstâncias. Qualquer situação cotidiana na qual faz-se necessário um julgamento moral, em que o agente moral necessita tomar decisões, se configura um problema ético e para tal abordagem pode-se arguir as teorias éticas, como é o caso da deliberação moral.

O método ou procedimento para análise bioética de conflitos éticos, proposto por Gracia, ${ }^{20}$ deve seguir os seguintes passos: a) apresentação do caso pela pessoa responsável pela tomada de decisão; b) discussão dos aspectos médicos da história clínica; c) identificação dos problemas morais que se apresentam; d) eleição por parte da pessoa responsável pelo caso, dos problemas morais envolvidos e com 
os quais se preocupa; e) identificação dos possíveis caminho; f) deliberação do melhor curso de ação; g) decisão final e h) argumentos contrários à decisão e argumentos contrários a esses argumentos que se disporia a defender publicamente.

Portanto, as relações interpessoais e profissionais constatadas no HC são fontes potenciais de conflitos éticos, inclusive com possíveis repercussões na qualidade do atendimento prestado aos pacientes.

A coalizão e os conflitos éticos que emergem nos cuidados em saúde não são sinais nem de inutilidade ética, nem de papel absoluto da ética nos cuidados em saúde. Decisões da sociedade e dos médicos são atingidas pelas políticas de saúde e pelas organizações de saúde, assim como pelo nível individual do pensamento ético. 21

O hospital escola é um espaço descrito como ambiente multiprofissional de ensino em serviço cuja finalidade é preparar profissionais para a realidade do sistema de saúde brasileiro. ${ }^{14}$

De acordo com as Diretrizes Curriculares Nacionais, os hospitais escolas são espaços pedagógicos para atividades práticas para os cursos da área da saúde, 22 e talvez por ter a diversidade de profissionais e por ser campo de práticas, seja local com alto potencial gerador de conflitos. Resta aprofundar a temática para definir com detalhes que os fatores favorecem e influenciam a geração de conflitos em hospital escola, na visão dos médicos e dos professores.

Médicos e enfermeiros deveriam estar alerta para a ocorrência de conflitos entre eles e, desde o processo de formação, deveriam ser preparados em atividades práticas cotidianas, sendo os conflitos resolvidos de maneira construtiva. ${ }^{23}$ A bioética, enquanto disciplina, desponta como uma possibilidade de instrumentalizar os profissionais de saúde para o enfrentamento dos conflitos éticos interrela- cional, seja com pacientes, profissionais da equipe e gestores, a nível pedagógico, profissional e político.

\section{Considerações finais}

Constata-se que na realidade do $\mathrm{HC}$, na perspectiva dos médicos, na relação entre o profissional de enfermagem e o médico no HC não há conflito consolidado, porém detecta-se existência de fatores geradores de conflito e que podem precipitá-lo, em situações reais. Esses achados não são apoiados pela literatura na qual predomina relatos os conflitos na relação.

Os fatores que favorecem a manutenção do equilíbrio são a comunicação e adequada divisão de funções entre as partes, a compreensão de cada profissional acerca de seu papel no cuidado com o paciente e a compreensão da importância da boa relação profissional para o cuidado do paciente. Por outro lado, as condições de trabalho e o fato de a instituição ser um hospital escola são aspectos favorecedores do conflito.

O conflito entre médico e enfermeiro pode ser um dos principais problemas nas instituições de saúde, já que entre eles se estabelece um vínculo profissional o mais estreito. Desse conflito, emergem problemas éticos que prejudicam a relação na equipe multidisciplinar, e, principalmente, o paciente. A depender dos resultados dessa relação, desse-se envidar esforços para que esses conflitos sejam evitados.

As informações obtidas a partir de contextos e perspectivas profissionais específicas tem inestimável valor, enquanto instrumento de avaliação e possível transformação da realidade apreendida, bem como, no delineamento de um horizonte ético para as relações profissionais em equipe multidisciplinar e seus reflexos sobre o bem-estar do paciente e a qualidade dos serviços em saúde.

\section{Referências}

1. Silva IMBP. A relação conflituosa entre médicos e enfermeiras no contexto hospitalar [tese]. São Paulo: Programa de Pós-graduação em Ciências Sociais da Pontífice Universidade Católica de São Paulo; 2006.

2. Lima IB, Bastos LO. Conflitos de poder na relação entre profissionais de saúde sob a óptica do paciente. Rev Enferm UFPE. 2007; 1: 19-27.

3. Silva ACC, Barros LCB, Barros CEC, Ferreira GE, Silva RF. Médicos e enfermeiras: o relacionamento numa unidade de emergência (UE). In: XXVI Encontro Nacional de Engenharia de Produção (ENEGEP); 9 a 11 out 2006; Fortaleza, CE; Anais do XXVI ENEGEP; Fortaleza:
ABEPRO; 2006. p. 1-9.

4. Araujo Netto LFS, Ramos FRS. Enfermeiro: o papel que se define nas relações conflituosas. In: 8o Simpósio Brasileiro de Comunicação em Enfermagem (SIBRACEn) [evento na internet]; 2 a 3 mai 2005; Ribeirão Preto, SP; Anais do 8० SIBRACEn; Ribeirão Preto; 2002. Disponível em: http://www.proceedings.scielo.br/scielo.php?script=sci_artt ext\&pid=MSC0000000052002000200058\&lng $=$ en\&nrm $=\mathrm{V}$ an.

5. Peduzzi M. Equipe multiprofissional de saúde: conceito e tipologia. Rev Saúde Pública. 2001; 35: 103-9. 
6. Pinho MCG. Trabalho em equipe de saúde: limites e possibilidades de atuação eficaz. Ciênc Cognição. 2006; 8: 6887.

7. Campos GWS. Reforma da reforma: repensando a saúde. São Paulo: Hucitec; 1992.

8. Stumm EMF, Maçalai RT, Kirchner RM. Dificuldades enfrentadas por enfermeiros em um centro cirúrgico. Texto \& Contexto Enferm. 2006; 35: 464-71.

9. Ribeiro EM, Pires D, Blank VLG. A teorização sobre processo de trabalho em saúde como instrumental para análise do trabalho no Programa de Saúde da Família. Cad Saúde Pública. 2004; 20: 438-46.

10. Zoboli ELCP. Bioética e atenção básica: um estudo de ética descritiva com médicos e enfermeiros do Programa de Saúde da Família [tese]. São Paulo: Programa de Pós-graduação da Faculdade de Sáude Pública da Universidade de São Paulo; 2003.

11. Brasil. Conselho Federal de Medicina. O médico e seu trabalho: aspectos metodológicos e resultados do Brasil. Brandão MC, Gouveia VV, coords. Brasilia, DF; 2004.

12. Stacciarini JMR, Andraus LMS, Esperidião E, Nakatan AK. Quem é o enfermeiro? Rev Eletrônica Enferm. [on line] 1999; 1 (1). Disponível em: http://www.revistas. ufg.br/índex.php/ fen/índex.

13. Mallory GA. Believe it or not: conflict can be healthy once you understand it and learn manage it. Nursing. 1981; 11: 97-102

14. Elias MA, Navarro VL. A relação entre o trabalho, a saúde e as condições de vida: negatividade e positividade no trabalho das profissionais de enfermagem de um hospital escola. Rev Latino-Am Enferm. 2006; 14: 517-25.
15. Oliveira AM, Lemes AM, Leão LR, Silva FL. Relação entre enfermeiros e médicos no Hospital das Clínicas da Universidade Federal de Goiás: a perspectiva do profissional de enfermagem. Comunicação oral. VIII Congresso Brasileiro de Bioética; 2009 Set 23-26; Búzios-Rio de Janeiro.

16. Fleury MTL. O desvendar da cultura de uma organização uma discussão metodológica. In: Fleury MTL, Fisher RM, orgs. Cultura e poder nas organizações. São Paulo: Atlas; 1989. p. 15-28.

17. Tabak N, Orit K. Relationship between how nurses resolve their conflicts with doctors, their stress and job satisfaction. J Nurs Manag. 2007; 15: 321-31.

18. Thomas EJ, Sexton JB, Helmreich RL. Discrepant attitudes about teamwork among critical care nurses and physicians. Crit Care Med. 2003; 31; 956-9.

19. Teixeira TC, Teixeira ML, Brodt SFM, Oliveira RP, Dexheimer Neto FL, Roehrig C, Oliveira ES. A adequada comunicação entre os profissionais médicos reduz a mortalidade no centro de tratamento intensivo. Rev Bras Terapia Intensiva. 2010; 22; 112-7.

20. Gracia D. La deliberación moral: El método de la ética clínica. Med Clin. 2001; 117; 18-23.

21. Vendemiatti M, Siqueira ES; Filardi F, Binotto E, Simioni FJ. Conflito na gestão hospitalar: o papel da liderança. Ciênc Saúde Coletiva. 2010; 15 (Supl. 1): 1301-14.

22. Brasil. Diretrizes curriculares nacionais dos cursos de graduação em medicina. Brasília, DF; 2001.

23. Serodio AMB, Almeida JAM. Situações de conflitos éticos relevantes para a discussão com estudantes de medicina: uma visão docente. Rev Bras Educ Méd. 2009; 33; 55-62.

Recebido em 29 de outubro de 2010

Versão final apresentada em 12 de novembro de 2010

Aprovado em 1 de dezembro de 2010 